\title{
ON THE EXISTENCE OF EIGENVALUES \\ OF DIFFERENTIAL OPERATORS DEPENDENT \\ ON A PARAMETER
}

BY

SH. STRELITZ AND S. ABRAMOVICH

\begin{abstract}
In this paper we obtain results about the existence of eigenvalues for a system which depends polynomially on $\lambda$,

$$
u_{k}^{\prime}(x)=\sum_{j=1}^{n} b_{k j}(x, \lambda) u_{j}(x), \quad \sum_{i=0}^{p} \sum_{j=1}^{N} a_{k j}^{i} u_{j}\left(x_{i}\right)=0,
$$

$k=1, \ldots, N$. In order to get these results we prove that this system can be reduced to a standard system of the form$$
y_{k}^{\prime}(x)=\sum_{j=1}^{n} a_{k j}(x, \lambda) y_{j}(x), \quad y_{k}(0)=a_{k}(\lambda), \quad y_{n}(1)=0,
$$

$k=1, \ldots, n$.
\end{abstract}

1. Introduction. The paper deals with the eigenvalue problem of the following system:

$$
\begin{gathered}
d U / d x=B(x, \lambda) U, \\
\sum_{i=0}^{p} A^{i} U\left(x_{i}\right)=0,
\end{gathered}
$$

where $U=\left(u_{1}, \ldots, u_{N}\right)^{T}, B(x, \lambda)$ is an $N \times N$ matrix, and its elements $b_{i j}(x, \lambda)$, $i, j=1, \ldots, N$, are polynomials in $\lambda$. The matrices $A^{i}, i=1, \ldots, p$, are of order $N \times N$ and are dependent on $\lambda$. The $\left\{x_{i}\right\}$ are $p+1$ given points in the segment $[0,1]$ and satisfy $0=x_{0} \leqslant x_{1} \leqslant x_{2} \leqslant \cdots \leqslant x_{p}$.

Eigenvalue problems have been the subject of numerous papers (for several of the first publications in the problem we investigate in this paper, see [4], [6]).

This work is a continuation and extension of [2], [8], [9].

In order to get the main result about the existence of the eigenvalues we will prove in $\$ 2$ a theorem which asserts that from the theoretical point of view there is only one type of system, which we call the standard system, that is, the system of the standard form:

$$
\begin{gathered}
d Y / d x=A(x, \lambda) Y, \quad 0<x<1, \\
Y(0)=a(\lambda), \\
y_{n}(1, \lambda)=0,
\end{gathered}
$$

Received by the editors June 26, 1978.

AMS (MOS) subject classifications (1970). Primary 34A10, 34B25; Secondary 30A64, 30 A84.

Key words and phrases. Eigenvalues, algebraic functions, order of entire functions, asymptotic expansion. 
where $Y=\left(y_{1}, \ldots, y_{n}\right)^{T}, A(x, \lambda)$ is an $n \times n$ matrix and its elements $a_{i j}(x, \lambda)$, $i, j=1, \ldots, n$, are polynomials in $\lambda$ and $a(\lambda)$ is a matrix of order $n \times 1$ dependent on $\lambda$, which is used instead of the wide class of systems of type (1.1) and (2.1).

2. The standard system. We are interested in systems dependent on a parameter, but, because in the following theorem it does not matter if such a parameter exists, for the sake of brevity the parameter will not appear explicitly. This theorem (Theorem 1) asserts that instead of (1.1), (2.1), it is sufficient to concentrate on the investigation of the main problem concerning the existence of the eigenvalues of the standard system (3.1), (4.1), (5.1).

THEOREM 1. Every system

$$
\begin{gathered}
U^{\prime}=B U, \quad 0 \leqslant x \leqslant 1, \\
\sum_{i=0}^{p} A^{i} U\left(x_{i}\right)=0,
\end{gathered}
$$

where $U=\left(u_{1}, \ldots, u_{N}\right)^{T}, B=\left\{b_{i j}\right\}, i, j=1, \ldots, N$, the $A^{i}=\left\{a_{k j}^{i}\right\}_{k j=1}^{N}, i=$ $0, \ldots, p$, are matrices of order $N \times N$ and $0 \leqslant x_{0}<x_{1}<\cdots<x_{p}$ are $p+1$ given points in the interval $[0,1]$, can be reduced to a standard system of the form

$$
\begin{gathered}
Y^{\prime}=A Y, \quad 0<x<1, \\
Y(0)=a, \\
y_{n}(1)=0,
\end{gathered}
$$

where $Y=\left(y_{1}, \ldots, y_{n}\right), A=\left\{a_{i j}\right\}, i, j=1, \ldots, n$, is a matrix of order $n \times n$ and $a$ is a matrix of order $n \times 1, n=\left({ }_{N}^{N(p+1)}\right)+1$. The system (1.2), (2.2) has $a$ nontrivial solution if and only if the standard system (3.2), (4.2), (5.2) has a solution.

Proof. Let us denote a fundamental system of solutions of (1.2) by $E(x)=\left\{e_{i j}\right\}$, $i, j=1, \ldots, n$, which satisfies the initial condition $E(0)=I=\left\{\delta_{i j}\right\}, i, j=$ $1, \ldots, n\left(\delta_{i j}\right.$ is the Kronecker symbol). Any solution $U(x)$ of (1.2) is a linear combination of the fundamental system of these solutions, that is,

$$
U(x)=E(x)\left(b_{1}, \ldots, b_{N}\right)^{T},
$$

where the $b_{i}, i=1, \ldots, N$, are constant.

By substituting $U(x)$ by $(6.2)$ in (2.2) we get

$$
\sum_{i=0}^{p} A^{i} E\left(x_{i}\right)\left(b_{1}, \ldots, b_{N}\right)^{T}=(0, \ldots, 0)^{T} .
$$

If there is a nontrivial solution of the system (1.2), (2.2), it is necessary that the determinant of the coefficients should vanish, that is

$$
D=\left|\sum_{i=0}^{p} A^{i} E\left(x_{i}\right)\right|=0 .
$$

(The determinant of a matrix $B$ is denoted by $|B|$.)

REMARK. In the case $B(x, \lambda)$, and thereby $E(x, \lambda)$, depends on a parameter $\lambda$, then $D$ depends on $\lambda$ too, and the eigenvalues, if any exist, are solutions of $D=0$. 
We intend to construct the new linear system (3.2), (4.2), (5.2) of which we have spoken above. We define $y_{n}(x)$ of (3.2), (4.2) by using (7.2) to be the following function:

$$
y_{n}(x)=\left|\sum_{i=0}^{p} A^{i} E\left(x x_{i}\right)\right| \text {. }
$$

It is obvious that if there is a solution of (1.2), (2.2) then $y_{n}(1)=D=0$ and

$$
y_{n}(0)=a_{n}=\left|\sum_{i=0}^{p} A^{i}\right|, \quad n=\left(\begin{array}{c}
N(p+1) \\
N
\end{array}\right)+1 .
$$

In the representation of $y_{n}(x)$ as the determinant $\left|\sum_{i=0}^{p} A^{i} E\left(x x_{i}\right)\right|$ the $j$ th column is $\sum_{i=0}^{p} A^{i} e_{j}\left(x x_{i}\right)$ where $e_{j}(x)$ is the $j$ th fundamental solution included in $E(x)$.

The determinant $y_{n}(x)=\left|\sum_{i=0}^{p} A^{i} E\left(x x_{i}\right)\right|$ can be expressed as a linear combination of determinants with coefficients constructed by the matrices $A^{k}, k=$ $0,1, \ldots, p$ :

$$
y_{n}(x)=\left|\sum_{i=0}^{p} A^{i} E\left(x x_{i}\right)\right|=\left|\left(A^{0}, A^{1}, \ldots, A^{p}\right)\left(E\left(x x_{0}\right), \ldots, E\left(x x_{p}\right)\right)^{T}\right|,
$$

where the components of the vectors $\left(A^{0}, A^{1}, \ldots, A^{p}\right)$ and $\left(E\left(x x_{0}\right), \ldots, E\left(x x_{p}\right)\right)$ are the matrices $A^{i}, i=0, \ldots, p$, and $E\left(x x_{i}\right), i=0, \ldots, p$, respectively, that means that $\left(A^{0}, A^{1}, \ldots, A^{p}\right)$ and $\left(E\left(x x_{0}\right), \ldots, E\left(x x_{p}\right)\right)$ are matrices of order $N \times N(p+1)$. Therefore by the Binet-Cauchy formula [3, p. 9]:

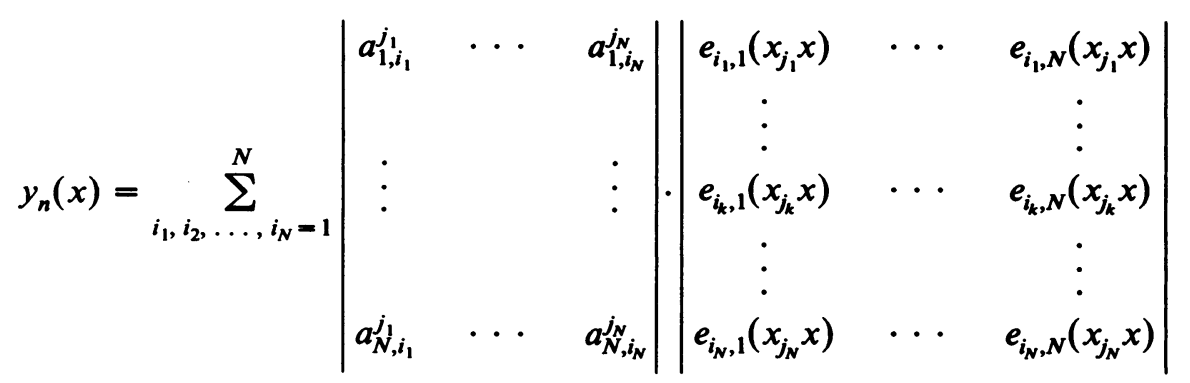

where $0<j_{1}<j_{2}<\cdots<j_{N}<p$, with $i_{k}<i_{k+1}$ if $j_{k}=j_{k+1}$.

A typical determinant included in the linear combination is as follows:

$$
\sigma=\left|\begin{array}{cccc}
e_{i_{1}, 1}\left(x_{j_{1}} x\right) & e_{i_{1}, 2}\left(x_{j_{1}} x\right) & \ldots & e_{i_{1}, N}\left(x_{j_{1}} x\right) \\
e_{i_{2}, 1}\left(x_{j_{2}} x\right) & \cdots & \ldots & e_{i_{2}, N}\left(x_{j_{2}} x\right) \\
\vdots & & & \vdots \\
e_{i_{k}, 1}\left(x_{j_{k}} x\right) & \ldots & \ldots & e_{i_{k}, N}\left(x_{j_{k}} x\right) \\
\vdots & & & \vdots \\
e_{i_{N}, 1}\left(x_{j_{N}} x\right) & \cdots & \ldots & e_{i_{N}, N}\left(x_{j_{N}} x\right)
\end{array}\right|
$$

where $i_{k}=1, \ldots, N, k=1, \ldots, N, 0<j_{1}<j_{2}<\cdots j_{N}<p$, with $i_{k}<i_{k+1}$ if $j_{k}=j_{k+1}$. 
We will show that the $\sigma$ determinants together with $y_{n}(x)$ form the standard system (3.2), (4.2), (5.2).

By differentiating such a determinant we obtain $N$ determinants in each of which one row is derivated and the other $N-1$ rows are the same as in the original determinant:

$$
\sigma^{\prime}=\left|\begin{array}{ccc}
e_{i_{1}, 1}\left(x_{j_{1}} x\right) & \cdots & e_{i_{1}, N}\left(x_{j_{1}} x\right) \\
\vdots & & \vdots \\
e_{i_{k}, 1}\left(x_{j_{k}} x\right) & \cdots & e_{i_{k}, N}\left(x_{j_{k}} x\right) \\
\vdots & & \vdots \\
e_{i_{N}, 1}\left(x_{j_{N}} x\right) & \cdots & e_{i_{N}, N}\left(x_{j_{N}} x\right)
\end{array}\right|=\sum_{k=1}^{N}\left|\begin{array}{ccc}
e_{i_{1}, 1}\left(x_{j_{1}} x\right) & \cdots & e_{i_{1}, N}\left(x_{j_{1}} x\right) \\
\vdots & & \vdots \\
e_{i_{k-1}, 1}\left(x_{j_{k-1}} x\right) & \cdots & e_{i_{k-1} N}\left(x_{j_{k-1}} x\right) \\
e_{i_{k}, 1}^{\prime}\left(x_{j_{k}} x\right) & \cdots & e_{i_{k}, N}^{\prime}\left(x_{j_{k}} x\right) \\
e_{i_{k+1}, 1}\left(x_{j_{k+1}} x\right) & \cdots & e_{i_{k+1}, N}\left(x_{j_{k+1}} x\right) \\
\vdots & & \vdots \\
\vdots & & \vdots \\
e_{i_{N}, 1}\left(x_{j_{N}} x\right) & \cdots & e_{i_{N}, N}\left(x_{j_{N}} x\right)
\end{array}\right|
$$

Obviously,

$$
e_{i_{k}, t}^{\prime}\left(x_{j_{k}} x\right)=x_{j_{k}} \sum_{r=1}^{N} b_{i_{k}, r} e_{r, t}\left(x_{j_{k}} x\right), \quad t=1,2, \ldots, N,
$$

where $e_{t}=\left(e_{1, t}(x), \ldots, e_{N, t}(x)\right)$ is the $t$ th fundamental solution of (1.2) included in $E(x)$.

Replacing $e_{i_{k}, t}^{\prime}\left(x_{j_{k}} x\right)$ according to (9.2) we get from (8.2):

$$
\sigma^{\prime}=\sum_{k=1}^{n} x_{j_{k}} \sum_{r=1}^{N} b_{i_{k}, r}\left|\begin{array}{ccc}
e_{i_{1}, 1}\left(x_{j_{1}} x\right) & \cdots & e_{i_{1}, N}\left(x_{j_{1}} x\right) \\
\vdots & & \vdots \\
e_{i_{k-1}, 1}\left(x_{j_{k-1}} x\right) & \cdots & e_{i_{k-1}, N}\left(x_{j_{k-1}} x\right) \\
e_{r, 1}\left(x_{j_{k}} x\right) & \cdots & e_{r, N}\left(x_{j_{k}} x\right) \\
e_{i_{k+1}, 1}\left(x_{j_{k+1}} x\right) & \cdots & e_{i_{k+1}, N}\left(x_{j_{k+1}} x\right) \\
\vdots & & \vdots \\
e_{i_{N}, 1}\left(x_{j_{N}} x\right) & \cdots & e_{i_{N}, N}\left(x_{j_{N}} x\right)
\end{array}\right|
$$

The absolute value of any determinant in the last summation (10.2) is already included in the family of determinants in which $\sigma$ is included, and so $\sigma^{\prime}$ is a sum of the determinants of type $\sigma$ with coefficients of the given system (1.2).

Therefore by appropriate numeration we get the new system (3.2), (4.2), (5.2).

Through the construction of the standard system (3.2), (4.2), (5.2) from the original system (1.2), (2.2) it is obvious that the existence of a nontrivial solution of the original system brings about the existence of a solution to the standard system. 
We will show that from the existence of a solution of the standard system, a nontrivial solution of (1.2), (2.2) follows: because of the identity

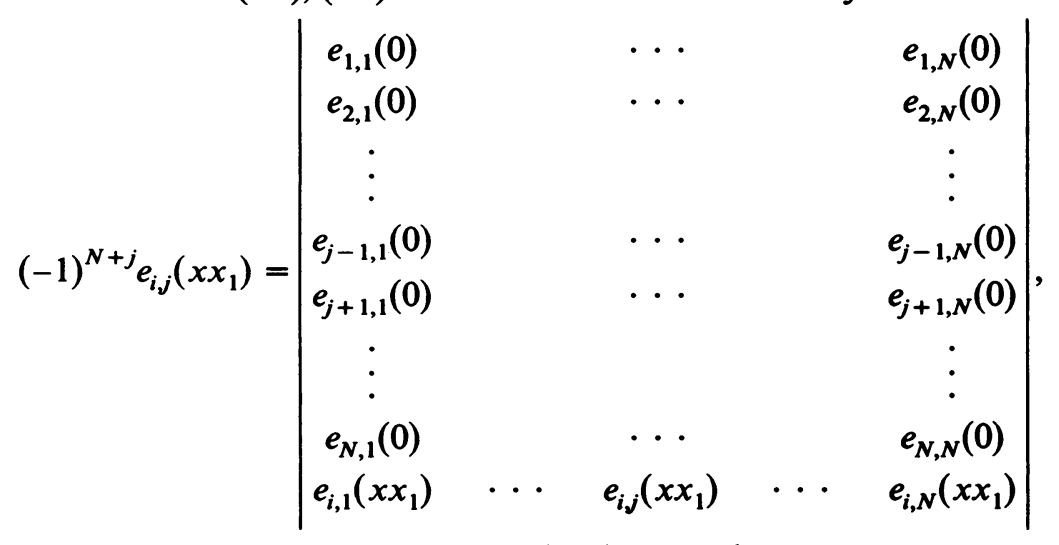

the standard system (3.2), (4.2), (5.2) includes the $N p$ subsystems

$$
\begin{aligned}
& e_{j}^{\prime}\left(x x_{k}\right)=x_{k} B e_{j}\left(x x_{k}\right), \quad e_{i, j}(0)=\delta_{i, j}, \\
& j=1, \ldots, N, \quad i=1, \ldots, N, \quad k=1, \ldots, p ;
\end{aligned}
$$

therefore, from the existence of the solution of (3.2), (4.2), (5.2) the existence of a nontrivial solution to (1.2), (2.2) follows, because, if the original system had only a trivial solution then $D \neq 0$, whereas from the existence of a solution to the standard system we get $y_{n}(1)=D=0$.

In order to demonstrate the procedure we will show explicitly how we build the standard system in two different cases:

(a) One scalar equation with $p+1$ points:

$$
\begin{gathered}
u^{\prime}(x)=b(x) u(x), \\
\sum_{i=0}^{p} a^{i} u\left(x_{i}\right)=0, \quad x_{0}=0<x_{1}<\cdots<x_{p}=1 .
\end{gathered}
$$

We denote by $e(x)$ the solution of (11.2) which satisfy $e(0)=1$. We define:

$$
\begin{gathered}
y_{i}(x)=e\left(x x_{i}\right), \quad i=1, \ldots, p \\
y_{p+1}(x)=\sum_{i=0}^{p} a^{i} e\left(x x_{i}\right)
\end{gathered}
$$

therefore

$$
y_{p+1}(x)=a^{0}+\sum_{i=1}^{p} a^{\dot{y}_{i}}(x),
$$

and the standard differential equation is

$$
\begin{aligned}
y_{i}^{\prime}(x) & =x_{i} b\left(x x_{i}\right) y_{i}(x), \quad i=1, \ldots, p, \\
y_{p+1}^{\prime}(x) & =\sum_{i=1}^{p} a^{i} x_{i} b\left(x x_{i}\right) y_{i}(x), \\
y_{i}(0) & =1, \quad i=1, \ldots, p \\
y_{p+1}(0) & =\sum_{i=0}^{p} a^{i}, \quad y_{p+1}(1)=0 .
\end{aligned}
$$


(b) A system with two equations with three boundary points:

$$
\begin{array}{cc}
u_{1}^{\prime}=u_{2}, & u_{2}^{\prime}=\lambda p u_{1}, \\
u_{1}(0)-u_{2}(0)=0, & u_{1}\left(\frac{1}{2}\right)-u_{2}(1)=0 .
\end{array}
$$

The standard system derived from (12.2), (13.2) includes 15 equations with 15 unknown functions, as we have shown above according to the Binet-Cauchy formula:

$$
\begin{aligned}
y_{15} & =\sum_{i=0}^{2} A^{i} E\left(x x_{i}\right) \\
& =\sum_{i_{1}, i_{2}=1}^{2}\left|\begin{array}{ll}
a_{1, i_{1}}^{j_{1}} & a_{1, i_{2}}^{j_{2}} \\
a_{2, i_{1}}^{j_{1}} & a_{2, i_{2}}^{j_{2}}
\end{array}\right| \cdot\left|\begin{array}{ll}
e_{i_{1}, 1}\left(x_{j_{1}} x\right) & e_{i_{1,2}}\left(x_{j_{1}} x\right) \\
e_{i_{2}, 1}\left(x_{j_{2}} x\right) & e_{i_{2}, 2}\left(x_{j_{2}} x\right)
\end{array}\right|
\end{aligned}
$$

where $0<j_{1}<j_{2} \leqslant 2$, with $i_{k}=1, i_{k+2}=2$ if $j_{1}=j_{2}$, or explicitly we get in this case

$$
\begin{aligned}
y_{15}= & 1\left|\begin{array}{ll}
e_{1,1}(0) & e_{1,2}(0) \\
e_{1,1}\left(\frac{1}{2} x\right) & e_{1,2}\left(\frac{1}{2} x\right)
\end{array}\right|-\left|\begin{array}{ll}
e_{2,1}(0) & e_{2,2}(0) \\
e_{1,1}\left(\frac{1}{2} x\right) & e_{1,1}\left(\frac{1}{2} x\right)
\end{array}\right| \\
& +\left|\begin{array}{ll}
e_{1,1}(0) & e_{1,2}(0) \\
e_{2,1}(x) & e_{2,2}(x)
\end{array}\right|-\left|\begin{array}{ll}
e_{2,1}(0) & e_{2,2}(0) \\
e_{2,1}(x) & e_{2,2}(x)
\end{array}\right|
\end{aligned}
$$

and the smallest subsystem which includes $y_{15}$ (the reduced system-see the next section) is

$$
\begin{array}{ll}
y_{1}^{\prime}=\frac{1}{2} y_{3}, & y_{1}(0)=0, \\
y_{2}^{\prime}=\frac{1}{2} y_{4}, & y_{2}(0)=-1, \\
y_{3}^{\prime}=\frac{1}{2} \lambda p y_{1}, & y_{3}(0)=1, \\
y_{4}^{\prime}=\frac{1}{2} \lambda p y_{2}, & y_{4}(0)=0, \\
y_{5}^{\prime}=y_{7}, & y_{5}(0)=0, \\
y_{6}^{\prime}=y_{8}, & y_{6}(0)=-1, \\
y_{7}^{\prime}=\lambda p y_{5}, & y_{7}(0)=1, \\
y_{8}^{\prime}=\lambda p y_{6}, & y_{8}(0)=0, \\
y_{15}^{\prime}=-\frac{1}{2} y_{3}+\frac{1}{2} y_{4}+\lambda p y_{5}-\lambda p y_{6}, & y_{15}(0)=0, y_{15}(1)=0,
\end{array}
$$

where

$$
\begin{aligned}
& y_{1}(x)=\left|\begin{array}{ll}
e_{1,1}(0) & e_{1,2}(0) \\
e_{1,1}(x / 2) & e_{1,2}(x / 2)
\end{array}\right|=e_{1,2}(x / 2), \\
& y_{2}(x)=\left|\begin{array}{ll}
e_{2,1}(0) & e_{2,2}(0) \\
e_{1,1}(x / 2) & e_{1,1}(x / 2)
\end{array}\right|=-e_{1,1}(x / 2), \\
& y_{3}(x)=\left|\begin{array}{ll}
e_{1,1}(0) & e_{1,2}(0) \\
e_{2,1}(x / 2) & e_{2,2}(x / 2)
\end{array}\right|=e_{2,2}(x / 2),
\end{aligned}
$$




$$
\begin{aligned}
& y_{4}(x)=\left|\begin{array}{ll}
e_{2,1}(0) & e_{2,2}(0) \\
e_{2,1}(x / 2) & e_{2,2}(x / 2)
\end{array}\right|=-e_{2,1}(x / 2), \\
& y_{5}(x)=\left|\begin{array}{ll}
e_{1,1}(0) & e_{1,2}(0) \\
e_{1,1}(x) & e_{1,2}(x)
\end{array}\right|=e_{1,2}(x), \\
& y_{6}(x)=\left|\begin{array}{ll}
e_{2,1}(0) & e_{2,2}(0) \\
e_{1,1}(x) & e_{1,2}(x)
\end{array}\right|=-e_{1,1}(x), \\
& y_{7}(x)=\left|\begin{array}{ll}
e_{1,1}(0) & e_{1,2}(0) \\
e_{2,1}(x) & e_{2,2}(x)
\end{array}\right|=e_{2,2}(x), \\
& y_{8}(x)=\left|\begin{array}{ll}
e_{2,1}(0) & e_{2,2}(0) \\
e_{2,1}(x) & e_{2,2}(x)
\end{array}\right|=-e_{2,1}(x) .
\end{aligned}
$$

3. The formulation of the main theorem. Theorem 1 in $\$ 2$ shows that it is sufficient to analyse only the standard problem considered in the previous section:

$$
\begin{gathered}
Y^{\prime}=A(x, \lambda) Y, \quad 0 \leqslant x<1, \\
Y(0)=a(\lambda), \\
y_{n}(1, \lambda)=0 .
\end{gathered}
$$

We want to find eigenvalues of (3.1), (4.1), (5.1) under the conditions that will be given in this chapter.

Definition 1.3. The reduced system. We construct the reduced system in the following way:

First step. We consider the system (3.1), (4.1), (5.1). If the coefficient matrix can be written as a direct sum of smaller matrices $A_{k}, k=1, \ldots, s$, we will concentrate only on investigating the subsystem connected to that $A_{k}$ which includes $y_{n}$ and removes the others.

Second step. Assume that (3.1), (4.1), (5.1) is the system we got after the first step. If the initial value problem implies that certain components are identically zero, then the equations of these components are eliminated. We continue this procedure until we get to a system that we cannot reduce by means of the two steps mentioned above. We call that system the reduced system. Thus it is obvious that in order to find eigenvalues of (3.1), (4.1), (5.1) it is sufficient to investigate only the reduced system. Therefore in the sequel, we assume that (3.1), (4.1), (5.1) is a reduced system.

The properties of the reduced system are of fundamental importance in this work, particularly in discussing the minorant.

The properties which play an important role are: (1) given a reduced system (3.1), (4.1), (5.1) where $A \geqslant 0, a \geqslant 0$, and where there is a segment $\left[\eta, x_{0}\right]<[0,1]$ and all the $a_{i j}(x, \lambda) \neq 0$ are positive for positive $\lambda$, then in $(\eta, 1), Y(x, \lambda)>0$ for $\lambda>0$; (2) under the same conditions, if for one $k$ at least $y_{k}(x, \lambda)>B e^{\beta \lambda^{\rho}\left(x-x_{0}\right)}$ then $y_{n}>B^{*} e^{\beta \lambda^{\rho}\left(x-x_{0}\right)}$, (see Lemma 3 and $\left.\$ 5\right)$.

The elements $a_{i j}(x, \lambda)$ of the matrix $A(x, \lambda)$ are polynomial in $\lambda$ :

$$
a_{i j}(x, \lambda)=a_{i j}^{1}(x) \lambda^{m_{i j}}+a_{i j}^{2}(x) \lambda^{m_{y}-1}+\ldots
$$


Using $a_{i j}^{1}(x)$ we define the following:

DEFINITION 2.3. We define

$$
\Delta\left(x_{0}, \lambda, \mu\right)=\operatorname{det}\left|-a_{i j}^{1}\left(x_{0}\right) \lambda^{m_{i j}}+\delta_{i j} \mu\right|_{i, j=1}^{n}=\mu^{n}+\sum_{k=0}^{n-1} p_{n-k}\left(x_{0}, \lambda\right) \mu^{k}
$$

where $p_{n-k}\left(x_{0}, \lambda\right)$ is a polynomial in $\lambda$ and $m_{k}$ denotes the degree of $p_{k}\left(x_{0}, \lambda\right)$.

Definition 3.3. We define $\rho=\max _{k=1, \ldots, n}\left(m_{k} / k\right)$ where $m_{k}$ is the degree of $p_{k}\left(x_{0}, \lambda\right)$.

In the neighbourhood of $\lambda=\infty$ the expansion of the solutions $\mu_{k}$ of $\Delta\left(x_{0}, \lambda, \mu\right)$ $=0$ has the following form:

$$
\mu_{k}=\beta_{k} \lambda^{p_{k} / q_{k}}\left[1+O\left(\lambda^{-1 / q_{k}}\right)\right], \quad k=1, \ldots, n,
$$

where $p_{k}$ and $q_{k}>0$ are integers;

$$
\rho=\max _{k=1, \ldots, n}\left(\frac{p_{k}}{q_{k}}\right)=\max _{k=1, \ldots, n}\left(\frac{m_{k}}{k}\right),
$$

where $m_{k}$ is the degree of $p_{k}\left(x_{0}, \lambda\right)$ (see [1, pp. 39-40, Theorem 15.2]).

Now we are ready to formulate our main theorem.

THEOREM 2. Let (3.1), (4.1), (5.1) be the reduced system. Suppose that:

(1) All the $a_{i j}^{k_{i j}+1}(x) \geqslant 0, k_{i j}=0,1, \ldots, m_{i j} ; i, j=1, \ldots, n$, are summable functions in $[0,1]$ and $a=\left(a_{1}, \ldots, a_{n}\right)^{T}$ is a nonnegative vector where each $a_{j}$ is $a$ polynomial in $\lambda$ with $|a|>0$.

(2) There is at least one point $x=x_{0}$ where all $a_{i j}(x, \lambda)$ are continuous functions (that means that for each pair $i, j$ there is a function of the equivalence class $a_{i j}(x, \lambda)$ which is continuous in $\left.x_{0}\right)$. At this point $x=x_{0}$ all the $a_{i j}(x, \lambda)($ of $(1.3))$ for positive $\lambda$ are positive for all the $i, j$ for which $a_{i j}(x, \lambda) \neq 0$.

(3) $\rho=\max _{k=1, \ldots, n}\left(m_{k} / k\right)$ is not an integer.

Under these conditions the problem (3.1), (4.1), (5.1) has an infinite sequence of eigenvalues.

REMARK 1.3. Working on the problem of the existence of eigenvalues of the system (3.1), (4.1), (5.1) in the case where the matrix $A$ may not be nonnegative, we have achieved results which, because of their length, will be published in a separate paper. These results are formulated in $\$ 11$.

REMARK 2.3. It is easy to verify that $a_{i j}(x, \lambda)$ will be always nonnegative for large enough $\lambda>0$ if $a_{i j}^{1}(x) \geqslant c>0, x \in[0,1]$.

REMARK 3.3. Theorem 2 can be proved for $-\infty<x<\infty$ because we can make a transformation from the real line to the interval $[0,1]$.

The following examples show that the conditions of Theorem 2 are essential.

EXAMPLE 1. Solving the system

$$
\begin{aligned}
y_{1}^{\prime} & =y_{2}, \quad y_{2}^{\prime}=y_{3}, \\
y_{3}^{\prime} & =-x\left(1+\left(20+x^{2}\right) \lambda\right) y_{2}+\left(2+\left(100+5 x^{2}\right) \lambda\right) y_{1}, \\
y_{1}(0) & =0, \quad y_{2}(0)=0, \quad y_{3}(1)=0,
\end{aligned}
$$

we get that $y_{3}=2+20 \lambda x^{3}$ has only one eigenvalue. 
This example stresses that condition (1) of Theorem 2 is essential.

EXAMPLE 2. The solution of the system

$$
\begin{aligned}
y_{1}^{\prime} & =b(x) \lambda^{m} y_{2}, \quad y_{2}^{\prime}=a(x) \lambda^{n} y_{1}, \\
y_{1}(0) & =a_{1}, \quad y_{2}(0)=a_{2}, \quad y_{1}(1)=0,
\end{aligned}
$$

where

$$
a(x)=\left\{\begin{array}{ll}
0, & 0 \leqslant x \leqslant \frac{2}{3}, \\
1, & \frac{2}{3}<x \leqslant 1,
\end{array} \quad b(x)= \begin{cases}1, & 0<x<\frac{1}{2}, \\
0, & \frac{1}{2}<x<1,\end{cases}\right.
$$

is $y_{1}=\frac{1}{2} \lambda^{n} a+a_{1}, \frac{1}{2} \leqslant x \leqslant 1$. Therefore, $y_{1}(1)=0$ has only a finite number of roots.

In the example, condition (2) is not fulfilled.

EXAMPLE 3. The system

$$
y_{1}^{\prime}=y_{2}, \quad y_{2}^{\prime}=\left(\frac{1}{2} \lambda^{2}-\lambda x\right) y_{2}+\left(\frac{1}{2} \lambda^{4}-\lambda^{3} x-\lambda\right) y_{1},
$$

has the solution $y_{1}=x e^{\lambda^{2} x}, y_{2}=\left(1+\lambda^{2} x\right) e^{\lambda^{2} x}$. Therefore the boundary value problem $y_{2}(0)=0, y_{1}(1)=0$ has no solutions. In this case $\rho=2$.

REMARK 4.3. The homogenous linear differential equation

$$
\frac{d^{n} y}{d x^{n}}=a_{1}(x, \lambda) \frac{d^{n-1} y}{d x^{n-1}}+\cdots+a_{n}(x, \lambda) y
$$

is equivalent to the homogenous linear system

$$
\begin{aligned}
& y_{1}^{\prime}=y_{2}, \\
& y_{2}^{\prime}=y_{3}, \\
& \vdots \\
& y_{n-1}^{\prime}=y_{n}, \\
& y_{n}^{\prime}=a_{n}(x, \lambda) y_{1}+a_{n-1}(x, \lambda) y_{2}+\cdots+a_{1}(x, \lambda) y_{n},
\end{aligned}
$$

where $y_{1}=y$ and $y_{k}=y^{(k-1)}$. Therefore we get an analogue to Theorem 2 for an $n$th order linear equation which is a generalisation of [2].

THEOREM 3. Under the same conditions of Theorem 2, there exist two positive constants $a>0, b>0$ which are independent of $j$ so that $a j^{1 / \rho}<\left|\lambda_{j}\right|<b j^{1 / \rho}$, $j=1,2, \ldots$

We get the same results of Theorems 2 and 3 if we replace (5.1) with $y_{n}(1, \lambda)=$ $a(1, \lambda)$ which is a transcendental entire function of order less than $\rho$.

4. Preliminary lemmas. If we prove that $y_{n}(1, \lambda)$ is an entire function of a noninteger order, then, as is known, the equation $y_{n}(1, \lambda)=0$ has an infinite sequence of solutions which are the eigenvalues of the system (3.1), (4.1), (5.1).

In order to find the order of $y_{n}(1, \lambda)$ we will build a majorant and a minorant of the solutions for a corresponding Cauchy problem. We will show that both the majorant $Z(x, \lambda)$ and the minorant $W(x, \lambda)$ are entire functions of $\lambda$, for each $x$, $\eta_{0}<x<1, \eta_{0} \geqslant 0, z_{n}(x, \lambda)$ and $w_{n}(x, \lambda)$ are of the same order $\rho$, from where we 
will conclude that $y_{n}(x, \lambda)$ of the Cauchy problem (3.1), (4.1) is also of the same order. Thereby the proof of Theorem 2 will be completed.

The construction of the majorant and the minorant system will be based on the following lemmas.

LEMMA 1. Let the systems

$$
y_{k}^{\prime}=\sum_{i=1}^{n} a_{k i}(x) y_{i}, \quad y_{k}(0)=a_{k} \geqslant 0, \quad k=1, \ldots, n,
$$

and

$$
z_{k}^{\prime}=\sum_{i=1}^{n} b_{k i}(x) z_{i}, \quad z_{k}(0)=b_{k}, \quad k=1, \ldots, n,
$$

satisfy the following conditions

$$
0 \leqslant a_{k i}(x) \leqslant b_{k i}(x), \quad a_{k} \leqslant b_{k}, \quad k, i=1, \ldots, n ;
$$

then, the solution $\left(y_{1}, \ldots, y_{n}\right)$ and $\left(z_{1}, \ldots, z_{n}\right)$ of the above systems satisfy $y_{n}(x)<$ $z_{k}(x), k=1, \ldots, n$.

Proof. As is well known, the Cauchy problems (1.4) and (2.4) can be solved by Piccard's successive approximations method (see, for example, [7]):

$$
\begin{aligned}
y_{k, m} & =a_{k}+\int_{0}^{x} \sum_{i=1}^{n}\left(a_{k i}(t) y_{i, m-1}\right) d t, \\
z_{k, m} & =b_{k}+\int_{0}^{x} \sum_{i=1}^{n}\left(b_{k i}(t) z_{i, m-1}\right) d t, \\
y_{k, 0} & =a_{k}, \quad z_{k, 0}=b_{k}, \quad k=1, \ldots, n, \quad m=1,2, \ldots,
\end{aligned}
$$

then the solutions will be, respectively, $y_{k}=\lim _{m \rightarrow \infty} y_{k, m}(x)$ and $z_{k}(x)=$ $\lim _{m \rightarrow \infty} z_{k, m}$. According to the conditions of the lemma, $y_{k, m}(x)<z_{k, m}(x)$; hence, $y_{k}(x)<z_{k}(x)$.

LEMMA 2. Suppose that the functions $a_{i j}(x, \lambda)$ and the vector $a$ of the Cauchy problem

$$
\begin{aligned}
& Y^{\prime}=A Y, \\
& Y(0)=a,
\end{aligned}
$$

satisfy the conditions of Theorem 2. Then, for every fixed $x, \operatorname{Max}_{|\lambda|=r}|Y(x, \lambda)|=$ $Y(x, r)$, and also $Y(x, r)$ is a nondecreasing vector in $x$ for each $r$.

Proof. By means of Piccard's successive approximation method we obtain

$$
\begin{aligned}
y_{k j}(x, \lambda)=a_{k}+\int_{0}^{x}\left[\sum_{e=0}^{m_{k 1}} a_{k 1}^{e+1}(t) \lambda^{m_{k, 1}-y_{1, j-1}}(t, \lambda)\right. \\
\\
\left.\quad+\cdots+\sum_{e=0}^{m_{k n}} a_{k n}^{e+1}(t) \lambda^{m_{k, n-} y_{n, j-1}(t, \lambda)}\right] d t
\end{aligned}
$$

for $j=1,2, \ldots, y_{k 0}(x)=a_{k}$. Here $y_{k j}(x, \lambda)$ is the $j$ th step in the successive approximation method of the $k$ th equation in the system (3.1), (4.1); therefore 
$y_{k, j}(x, \lambda)$ is a polynomial in $\lambda$ with nonnegative coefficients:

$$
y_{k j}(x, \lambda)=\sum_{h=0}^{\nu_{k j}} p_{h j}(x) \lambda^{h}, \quad p_{h j}(x) \geqslant 0, \quad k=1, \ldots, n, j=0,1, \ldots
$$

Thus,

$$
\operatorname{Max}_{|\lambda|=r}\left|y_{k j}(x, \lambda)\right| \leqslant \sum_{h=0}^{\nu_{k j}} p_{h j}(x) r^{h}=y_{k j}(x, r), \quad k=1, \ldots, n, j=0,1, \ldots
$$

The sequence $y_{k j}(x, \lambda), j=0,1, \ldots$, converges uniformly on the set $|\lambda|<R$ for each $R<\infty$ to $y_{k}(x, \lambda)$ and from (4.4) we now obtain that

$$
\operatorname{Max}_{|\lambda|=r}\left|y_{k}(x, \lambda)\right|=y_{k}(x, r), \quad k=1,2, \ldots, n .
$$

The equalities (3.4) also show that $y_{k}(x, r)$ is a nondecreasing function in $x$ for each $r$.

Relying upon these two lemmas we intend to estimate the order of the solution $Y(x, \lambda)$ of $(3.1),(4.1)$. Towards that end we will construct a majorant $Z(x, \lambda)$ and a minorant $W(x, \lambda)$ to $Y(x, \lambda)$, and show that both are of order $\rho$.

5. The construction of the majorant. We return now to the problem

(3.1) $Y^{\prime}=A Y$,

(4.1) $Y(0)=a$.

First of all we show that without loss of generality we can assume that under the hypothesis of Theorem $2, A(x, \lambda)$ for a fixed $\lambda$ is a bounded matrix.

Recalling that the elements $a_{i j}(x, \lambda)$ of the matrix $A(x, \lambda)$ are polynomials in $\lambda$ :

$$
a_{i j}(x, \lambda)=a_{i j}^{1}(x) \lambda^{m_{i}}+a_{i j}^{2}(x) \lambda^{m_{i j}-1}+\ldots
$$

let us define

$$
\beta(x)=\sup \left\{a_{i j}^{l}(x)\right\}, \quad 0<x \leqslant 1, \quad i, j=1, \ldots, n, \quad l=1, \ldots, m_{i j}+1,
$$

which evidently is summable. Then the matrix

$$
\tilde{A}(x, \lambda)=\frac{A(x, \lambda)}{k(\beta(x)+1)}
$$

is bounded, and $Y^{\prime}=k(\beta(x)+1) \tilde{A}(x, \lambda) Y$, where the number $k$ will be defined later.

Transforming the independent variable $x$ into $t: x \stackrel{\phi}{\rightarrow} t$,

$$
\phi: t=k \int_{0}^{x}(\beta(s)+1) d s
$$

we get a new system:

$$
\begin{gathered}
\frac{d Y^{*}}{d t}=A^{*}(t, \lambda) Y^{*}, \quad Y^{*}(0)=a, \\
Y^{*}(t)=Y(x(t)), \quad A^{*}(t, \lambda)=\tilde{A}(x(t), \lambda),
\end{gathered}
$$

with bounded matrix $A^{*}$, where $k$ is chosen so that $[0,1] \stackrel{\phi}{\rightarrow}[0,1]$. So we can assume that the original matrix $A(x, \lambda)$ is bounded for every fixed $\lambda$. 
We can find $c_{i j}>0$ such that

$$
\lambda^{m_{i j}} \sum_{e=0}^{m_{i j}} a_{i j}^{1+e}(x) \lambda^{-e} \leqslant c_{i j} \lambda^{m_{i j}}
$$

holds. Then, according to Lemma 1, the system

$$
z_{i}^{\prime}(x, \lambda)=\sum_{j=1}^{n} c_{i j} \lambda^{m_{i j}} z_{j}(x, \lambda), \quad i=1,2, \ldots, n,
$$

will be a majorant system of (3.1). Because of the nonnegativity of the values $C=\left\{c_{i j} \lambda^{m_{i j}}\right\}, i, j=1, \ldots, n$, the greatest characteristic root is nonnegative; this means that every other root has an absolute value not larger than the greatest characteristic value.

After we have defined the matrix $C$, the characteristic values $\mu_{k}$ have to be calculated from the following determinant:

$$
\Delta_{0}(\lambda, \mu, C)=\operatorname{det}\left|\left(c_{i j} \lambda^{m_{i j}}-\delta_{i j} \mu\right)\right|_{i, j=1}^{n}=0 .
$$

Therefore, in the neighbourhood of $\lambda=\infty$,

$$
\mu_{k}=\beta_{k}^{*} \lambda^{p_{k} / q_{k}}\left[1+O\left(\lambda^{-1 / q_{k}}\right)\right]
$$

$q_{k}>0, p_{k}$ are integers.

For every $\mu_{k}$ there is a solution $z=\left(z_{1}(x, \lambda), \ldots, z_{n}(x, \lambda)\right)=$ $\left(A_{1} e^{\mu x}, \ldots, A_{n} e^{\mu x}\right)$ in which the coefficients $A_{k}$ are computed from equation (1.5) and are polynomials in $x$ and rational functions of $\lambda$ and $\mu$. Any solution of (3.5) is a combination of the type

$$
z_{j}(x, \lambda)=\sum_{k=1}^{n} b_{k} A_{k j} e^{\mu_{k} x}, \quad j=1, \ldots, n,
$$

where $b_{k}=b_{k}(\lambda), k=1, \ldots, n$, are determined uniquely by the initial conditions, and therefore of the same type as the $A_{k j}$ th, that is, the $b_{k}$ th are rational functions of $\lambda$ and $\mu$ too.

So from all that has been explained above together with Lemmas 1 and 2 we conclude that

$$
\begin{aligned}
M\left(r, y_{j}\right) & =\max _{|\lambda|=r}\left|y_{j}(x, \lambda)\right|=y_{j}(x, r)<z_{j}(x, r) \\
& \leqslant \sum_{k=1}^{n}\left|b_{k}\right| A_{j k} e^{\mu_{k} x}<B r^{\rho_{0}} \exp \left(\beta_{0} r^{\rho_{1}} x\right)
\end{aligned}
$$

where $B, \rho_{1}$ and $\beta_{0}$ are numbers which are independent of $j$. We had defined $\rho$ in Definition 3.3 as $\rho=\max _{k=1, \ldots, n}\left(m_{k} / k\right)$ in the expansion of $\Delta\left(x_{0}, \lambda, \mu\right)$ in (2.3). Through the fact that in the development of $\mu_{k}$ in (3.3)

$$
\max _{k=1, \ldots, n}\left(p_{k} / q_{k}\right)=\rho_{1},
$$

we get

$$
y_{j}(x, r)=M\left(r, y_{j}\right)<B r^{\rho_{0}} \exp \left(\beta_{0} r^{\rho_{1}} x\right), \quad j=1, \ldots, n,
$$

that is, $y_{j}(x, \lambda), j=1, \ldots, n$, are entire functions of order not higher than $\rho_{1}$. 
6. Simplification of the problem. In this chapter we intend to clarify some circumstances that will simplify the construction of the minorant system of (3.1), (4.1).

Let $\left[\eta, x_{0}\right.$ ] be a segment where all the $a_{i j}^{1}(x) \neq 0$ are positive and continuous except perhaps in a set of measure zero (see the hypothesis of Theorem 2). Let $\eta_{0}=\left(\eta+x_{0}\right) / 2$.

In the sequel we need the following:

LEMMA 3. Under the hypothesis of Theorem 2, the solution of the Cauchy problem (3.1), (4.1) satisfies the inequality $Y\left(\eta_{0}, \lambda\right)>0$.

Proof. The matrix $A$ is nonnegative and $Y(0)=a \geqslant 0$. By comparing the system (3.1), (4.1) with the system $Y^{\prime}(x)=0, Y(0)=a$, we obtain in accordance with Lemma 1 that $Y(x, \lambda) \geqslant 0$ for $x \in[0,1]$. Moreover, with no loss of generality we can assume for simplicity that $1,2, \ldots, q$ are the indices for which $a_{j}>0$, then according to Lemma $1, y_{1}(x), y_{2}(x), \ldots, y_{q}(x)$, are positive in the interval $[0,1]$. We now choose all the equations in which at least one function of the set $y_{1}$, $y_{2}, \ldots, y_{q}$ appears on their right side. Suppose those are the equations with the indices $m_{1}, m_{2}, \ldots, m_{p}$. By using $A(x, \lambda) \geqslant 0$ and $Y(x, \lambda) \geqslant 0$ we obtain that for the indices $\nu=m_{1}, m_{2}, \ldots, m_{p}, y_{\nu}^{\prime}(x, \lambda)>0$ in the segment $\left[\eta_{0}, x_{0}\right]$. Therefore, because of (4.1), $y_{\nu}(x, \lambda)>0, \nu=m_{1}, m_{2}, \ldots, m_{p}$. Repeating this process, we get after a finite number of steps that all $y_{\nu}\left(\eta_{0}, \lambda\right)>0, \nu=1, \ldots, n$, otherwise the system (3.1), (4.1) was not in a reduced form in the sense of Definition 1.3.

Let us discuss the system (3.1), (4.1) again. By means of permutation we can put the matrix $A$ of (3.1) in the normal triangular form:

$$
A=\left(\begin{array}{lllllll}
A_{1} & 0 & 0 & 0 & 0 & 0 & 0 \\
\cdot & A_{2} & \cdot & \cdot & \cdot & \cdot & \cdot \\
0 & 0 & A_{g} & 0 & 0 & 0 & 0 \\
A_{g+1,1} & A_{g+1,2} & A_{g+1, g} & A_{g+1} & 0 & 0 & 0 \\
\vdots & & & & A_{g+2} & 0 & 0 \\
A_{s, 1} & \vdots & & & & \vdots & \vdots \\
A_{s, 2} & & \cdots & & A_{s, s-1} & A_{s}
\end{array}\right) ;
$$

here $A_{1}, A_{2}, \ldots, A_{s}$ are square irreducible matrices and in each row at least one matrix $A_{f, 1}, \ldots, A_{f, f-1}, f=g+1, \ldots, s$, is different from zero [3]. (A square matrix $A=\left\{a_{i_{k}}\right\}_{i, k=1, \ldots, n}$ is called irreducible if the index set $1,2, \ldots, n$ cannot be split into two complementary sets with common indices $i_{1}, i_{2}, \ldots, i_{\mu} ; k_{1}$, $k_{2}, \ldots, k_{\nu}, \mu+\nu=n$, such that $a_{i_{\alpha} k_{\beta}}=0, \alpha=1,2, \ldots, \mu ; \beta=1,2, \ldots, \nu$.)

We would like to point out that we have already dissected the original system into subsystems in such a way that taking into consideration the initial condition (4.1), we have built $A$ to be the smallest subsystem which includes $y_{n}$ and can be solved separately. 
Now we come back to the "characteristic equation" of (3.1).

$$
\begin{aligned}
\Delta\left(x_{0}, \lambda, \mu\right) & =\operatorname{det}\left|-a_{i j}^{1}\left(x_{0}\right) \lambda^{m_{y}}+\delta_{i j} \mu\right|_{i, j-1}^{n}=\mu^{n}+\sum_{k=0}^{n-1} p_{n-k}\left(x_{0}, \lambda\right) \mu^{k} \\
& =\mu^{n}+\sum_{k=1}^{n-1} p_{n-k}\left(x_{0}, \lambda\right) \mu^{k} .
\end{aligned}
$$

Using the normal form of $A$ (1.6), we get that

$$
\Delta\left(x_{0}, \lambda, \mu\right)=\prod_{i=1}^{s}\left|-A_{i}+I \mu\right|=\prod_{i=1}^{s} \Delta_{i}\left(x_{0}, \lambda, \mu\right) .
$$

In the neighbourhood of $\lambda=\infty$ we get that the expansion of the solutions $\mu_{k}$, $k=1, \ldots, n$, have the form (3.3). The maximal characteristic value of the expansion of $\Delta\left(x_{0}, \lambda, \mu\right)=0, \mu_{1}$ is a solution of at least one of the subequations

$$
\left|-A_{l}+I \mu\right|=\mu^{n_{l}}+\sum_{k=0}^{n_{l}-1} \tilde{p}_{n_{l}-k}\left(x_{0}, \lambda\right) \mu^{k}=0
$$

where $\tilde{p}_{n_{l}-k}\left(x_{0}, \lambda\right)$ is a polynomial in $\lambda$. We denote $\tilde{m}_{k}$ to be the degree of $\tilde{p}_{k}\left(x_{0}, \lambda\right)$. Similarly to the indicated solutions, the expansion of the solutions of $\left|-A_{l}+I \mu\right|=$ 0 in the neighbourhood of $\lambda=\infty$ is

$$
\mu_{k}=\tilde{\beta} \lambda^{\tilde{p}_{k} / \tilde{q}_{k}}\left[1+O\left(\lambda^{-1 / \tilde{q}_{k}}\right)\right], \quad \tilde{q}_{k}>0, \quad \tilde{p}_{k} \text { are integers, } \quad k=1, \ldots, n_{l} .
$$

The same considerations as before brings us the conclusion that

$$
\rho=\max _{k=1, \ldots, n_{t}-1}\left(\frac{\tilde{m}_{k}}{k}\right)=\max _{k=1, \ldots, n_{l}}\left(\frac{\tilde{p}_{k}}{\tilde{q}_{k}}\right) .
$$

In accordance with the dissection of $A$ into blocks we also dissect the columns $Y$ and $a$ into parts $Y^{k}, a^{k}, k=1, \ldots, s$,

$$
\begin{aligned}
& Y=\left(y_{1}, \ldots, y_{n}\right)=\left(y_{1}^{1}, \ldots, y_{n_{1}}^{1} ; \ldots ; y_{1}^{k}, \ldots, y_{n_{k}}^{k} ; \ldots ; y_{1}^{s}, \ldots, y_{n_{3}}^{s}\right), \\
& a=\left(a_{1}, \ldots, a_{n}\right)=\left(a_{1}^{1}, \ldots, a_{n_{1}}^{1} ; \ldots ; a_{1}^{k}, \ldots, a_{n_{k}}^{k} ; \ldots ; a_{1}^{s}, \ldots, a_{n_{3}}^{s}\right) .
\end{aligned}
$$

We will show that if $y_{k}^{\prime}(x, \lambda)>B e^{\beta \lambda^{\rho}\left(x-x_{0}\right)}, k=1, \ldots, n_{l}$, then $y_{n}>B e^{\beta \lambda^{\rho}\left(x-x_{0}\right)}$, where $B, \beta$ are constants.

Lemma 3 states that under the hypothesis of Theorem $2, y_{1}\left(\eta_{0}\right), \ldots, y_{n}\left(\eta_{0}\right)$ are positive; therefore, if we choose $b_{1}, \ldots, b_{n}$ to be sufficiently small numbers, the 
system

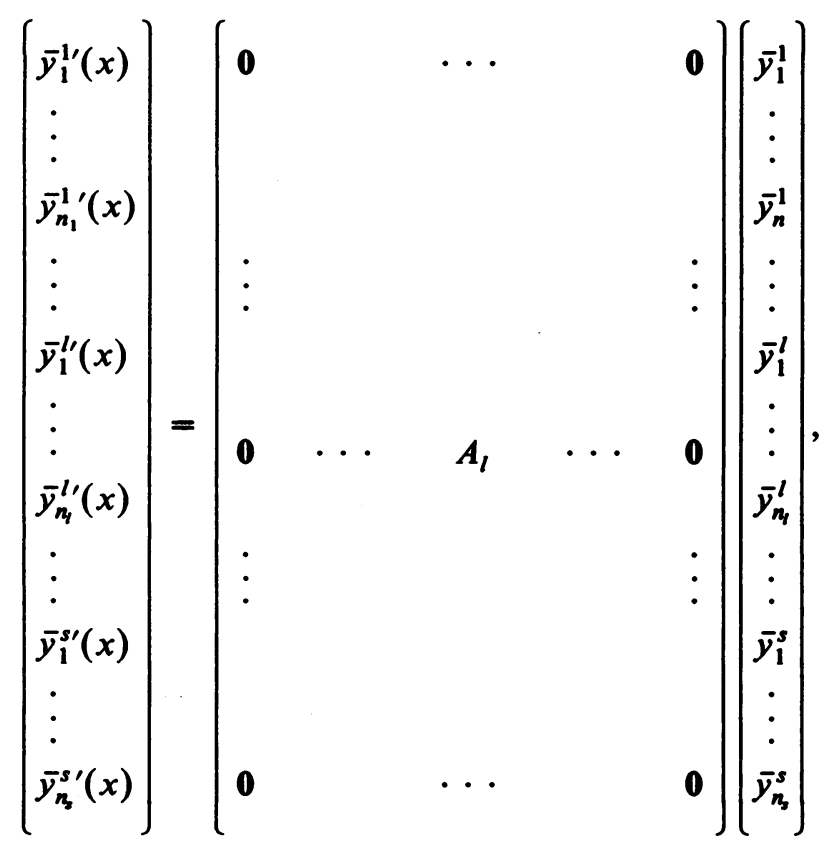

where 0 represents a submatrix with all entries 0 ,

$$
\left(\begin{array}{l}
\bar{y}_{1}^{1}\left(\eta_{0}\right) \\
\vdots \\
\bar{y}_{n_{1}}^{1}\left(\eta_{0}\right) \\
\vdots \\
\bar{y}_{1}^{l}\left(\eta_{0}\right) \\
\vdots \\
\bar{y}_{n_{l}}^{l}\left(\eta_{0}\right) \\
\vdots \\
\bar{y}_{1}^{s}\left(\eta_{0}\right) \\
\vdots \\
\bar{y}_{n_{3}}^{s}\left(\eta_{0}\right)
\end{array}\right)=\left(\begin{array}{c}
0 \\
\vdots \\
\vdots \\
b_{1}^{l} \\
\vdots \\
\vdots \\
\vdots \\
0
\end{array}\right)
$$

is a minorant system of (3.1) with the initial condition

$$
y_{i}\left(\eta_{0}\right)=a_{i}\left(\eta_{0}\right), \quad i=1, \ldots, \eta,
$$

where the $a_{i}\left(\eta_{0}\right)$ are the positive values that we have obtained at $x=\eta_{0}$ when we solved the system (3.1), (4.1). 
In order to solve the system (2.6), (3.6) it is enough to solve the system $\bar{Y}^{\prime \prime}=A_{l} Y^{l}, \bar{Y}^{\prime}\left(\eta_{0}\right)=b^{l}$. Indeed, suppose that the $\bar{y}_{k}^{l}(x, \lambda), k=1, \ldots, n_{l}$, are bounded from below in the following way:

$$
\bar{y}_{k}^{l}(x, \lambda) \geqslant\left\{\begin{array}{ll}
B \lambda^{\beta} e^{\omega \lambda^{\rho}\left(x-\eta_{0}\right)}, & x \in\left[\eta_{0}, x_{0}\right], \\
B \lambda^{\beta} e^{\omega \lambda^{\rho}\left(x_{0}-\eta_{0}\right)}, & x \in\left[x_{0}, 1\right],
\end{array} \quad k=1, \ldots, n_{l},\right.
$$

and the other functions $\bar{y}_{j}(x, \lambda)$ of the solution of (2.6), (3.6) are equal to zero, then since the system (2.6), (3.6) is a minorant system to (3.1), (4.6) (which is the system (3.1), (4.1) restricted to the segment $\left.\left[\eta_{0}, 1\right]\right)$, and the elements $A(x, \lambda)$ are polynomials in $\lambda$ therefore the function $y_{k}(x, \lambda), k=1, \ldots, n$, of the original system (3.1), (4.1) satisfy the inequalities

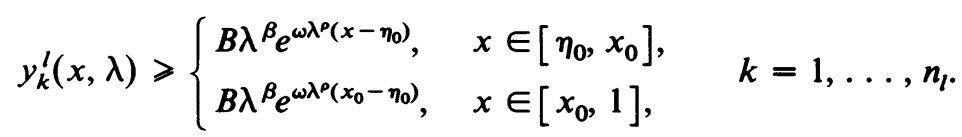

In order to find a lower bound for $y_{n}(x, \lambda)$ we choose all the equations in (3.1) in which $y_{1}, \ldots, y_{n}$ appear on their right side explicitly. Suppose those are the equations with the indices $p_{1}, p_{2}, \ldots, p_{q}$; then, since $A(x, \lambda)$ is a nonnegative matrix with polynomial elements in $\lambda$ we get that

$$
y_{\nu}^{\prime} \geqslant(\text { polynomial in } \lambda) \cdot B \lambda e^{\omega \lambda^{\rho}\left(x-\eta_{0}\right)}
$$

and together with $y_{\nu}\left(\eta_{0}\right) \geqslant 0, \nu=p_{1}, p_{2}, \ldots, p_{q}$, we get again

$$
y_{\nu}(x, \lambda) \geqslant\left\{\begin{array}{ll}
B_{\nu} \lambda^{B_{r}} e^{\omega \lambda^{\rho}\left(x-\eta_{0}\right)}, & x \in\left[\eta_{0}, x_{0}\right], \\
B_{\nu} \lambda^{\beta_{r}} e^{\omega \lambda^{\rho}\left(x_{0}-\eta_{0}\right)}, & x \in\left[x_{0}, 1\right],
\end{array} \quad \nu=p_{1}, p_{2}, \ldots, p_{q} .\right.
$$

The system (3.1), (4.1) is a reduced system (see Definition 1.3); therefore, by repeating this procedure a finite number of steps we get at last that:

$$
y_{n}(x, \lambda) \geqslant \begin{cases}B_{n} \lambda^{\beta_{n}} e^{\omega \lambda^{\rho}\left(x-\eta_{0}\right)}, & x \in\left[x_{0}, \eta_{0}\right], \\ B_{n} \lambda^{\beta_{n}} e^{\omega \lambda^{\rho}\left(x_{0}-\eta_{0}\right)}, & x \in\left[\eta_{0}, 1\right] .\end{cases}
$$

Thus, it is enough to prove the inequality (5.6) for the case that $A(x, \lambda)$ is itself an irreducible matrix.

7. The construction of the minorant. We explained at the end of $\$ 6$ that it is enough to construct a minorant in the case where $A$ is an irreducible matrix.

We construct the minorant in such a way that the order of its solution is $\rho$ too (see Definitions 2.3 and 3.3 of $\$ 3$ ).

We have defined in Definition 2.3:

$$
\Delta\left(x_{0}, \lambda, \mu\right)=\operatorname{det}\left|-a_{i j}^{1}\left(x_{0}\right) \lambda^{m_{i j}}+\delta_{i j} \mu\right|_{i, j=1}^{n}=\mu^{n}+\sum_{k=0}^{n-1} p_{n-k}\left(x_{0}, \lambda\right) \mu^{k} .
$$

$p_{n-k}\left(x_{0}, \lambda\right) \mu^{k}$ is obtained by decomposing $\Delta\left(x_{0}, \lambda, \mu\right)$, and $p_{n-k}\left(x_{0}, \lambda\right)$ is thus a sum of products of factors $a_{i j}^{1}\left(x_{0}\right) \lambda^{m_{\nu}}$. If $m_{k}$ is the degree of $p_{k}\left(x_{0}, \lambda\right)$, then $\rho=\max _{k=1, \ldots, m}\left(m_{k} / k\right)=m_{\bar{k}} / \bar{k}$ (there may be several indices $\bar{k}$; we choose one of them). 
It may occur that some of the monoms of the expansion of the determinant whose sums are equal cancel each other. We define $m_{k}^{0}$ to be the highest degree in $\lambda$ of these monoms which make up $p_{k}\left(x_{0}, \lambda\right), k=1, \ldots, n-1$, before the cancellation of equal monoms and $\rho_{0}=\max _{k=1, \ldots, n}\left(m_{k}^{0} / k\right)=m_{k_{1}}^{0} / k_{1}$. It is obvious that ${ }^{1}$

$$
\rho_{0}>\rho_{1} \text {. }
$$

Let

$$
D^{*}=a_{i_{1} j_{1}}^{1} \lambda^{m_{i j} a_{i_{2} j_{2}}^{1}} \lambda^{m_{i_{j} j_{2}}}, \ldots, a_{i_{q} j_{q}}^{1} \lambda^{m_{i q_{q}}}
$$

be the monom of order $m_{k_{1}}^{0}$ in $\lambda$.

We construct the minorant system only in the segment $\left[\eta_{0}, 1\right]$ because we are interested in the behavior of the system (3.1), (4.1) near $x=1$, therefore we put the initial value in $x=\eta_{0}$.

Now we build the minorant system

$$
w_{i_{k}}^{\prime}(x, \lambda)=d_{i_{k} j_{k}} \lambda^{m_{i k k} w_{j_{k}}}(x, \lambda), \quad k=1, \ldots, q, \quad x \in\left[\eta_{0}, 1\right],
$$

in the following way

$$
d_{i_{k} j_{k}}=\left\{\begin{array}{ll}
\min \left(a_{i_{k} j_{k}}^{1}(x)\right), & x \in\left[\eta_{0}, x_{0}\right], \\
0, & x \notin\left[\eta_{0}, x_{0}\right],
\end{array} \quad k=1, \ldots, q,\right.
$$

and we denote $m_{i_{k} j_{k}}=m_{i_{k}}, d_{i_{k} j_{k}}=d_{i_{k}}$. We get

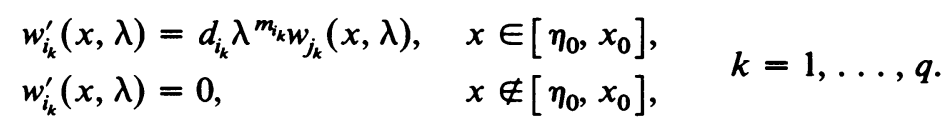

Since we are interested only in what happens in $\left[\eta_{0}, 1\right]$ we choose the initial condition to be

$$
w_{i_{k}}^{\prime}\left(\eta_{0}, \lambda\right)=b_{i_{k}}, \quad k=1, \ldots, q,
$$

where the $b_{i_{k}}$ are positive numbers small enough to be less than $y_{i_{k}}\left(\eta_{0}, \lambda\right)=$ $a_{i_{k}}\left(\eta_{0}\right)$-the solutions of (3.1), (4.1) at the point $x=\eta_{0}$ (which according to Lemma 3 are positive numbers).

The same reasoning as that of $\$ 6$ leads to the conclusion that for estimation from below of the solution $y_{n}$ of (3.1), (4.1) it is enough to estimate from below the solution of (2.7), (3.7).

For the sake of simplicity we rearrange the system (2.7), (3.7) and bring it to the form

$$
\begin{array}{ll}
w_{i}^{\prime}=d_{i} \lambda{ }^{m_{i}} w_{j_{i}}(x, \lambda), & x \in\left[\eta_{0}, x_{0}\right], \\
w_{i}^{\prime}(x, \lambda)=0, & x \notin\left[\eta_{0}, x_{0}\right], \\
w_{i}\left(\eta_{0}, \lambda\right)=b_{i}, & i=1, \ldots, q .
\end{array}
$$

\footnotetext{
${ }^{1}$ At the end of the next chapter we will get the conclusion that $\rho_{0}=\rho$. It is obvious that when $\rho_{0}=\rho$ there is at least one index $\bar{k}$ such that $k_{1}=\bar{k}$ and $m_{k_{1}}^{0}=m_{\bar{k}}$. It means, that at least one of the monoms with the highest fraction $m_{k_{1}}^{0} / k_{1}$ is not cancelled. That is a result which has nothing to do with the order of entire functions but is a result in connection with determinant expansion.
} 
8. Investigating the solutions of (4.7), (5.7). The characteristic equation of (4.7) in $x \in\left[\eta_{0}, x_{0}\right]$ is $\mu^{q}+\cdots+B_{q} \lambda^{l q}=0$, and because of the structure of (4.7), $\rho_{0}=$ $l_{q} / q$. If the system (4.7) can be divided into $s$ subsystems then

$$
\mu^{q}+\cdots+B_{q} \lambda^{l q}=\prod_{j=1}^{s}\left(\mu^{k_{j}}-\Delta_{j} \lambda^{p_{j}}\right)
$$

where

$$
\begin{aligned}
p_{j} & =\sum_{i=q_{j}+1}^{q_{j+1}} m_{i}, \quad \Delta_{j}=\sum_{i=q_{j}+1}^{d_{j+1}} d_{i}, \\
k_{j} & =q_{j+1}-q_{j}, \quad j=1, \ldots, s, \\
q & =k_{1}+\cdots+k_{s}, \quad l_{q}=p_{1}+\cdots+p_{s}, \\
\rho_{0} & =\left(p_{1}+\cdots+p_{s}\right) /\left(k_{1}+\cdots+k_{s}\right) .
\end{aligned}
$$

LEMMA 4. If the system (4.7) can be divided into $s$ subsystems then $p_{i} / k_{i}=\rho_{0}$, $i=1, \ldots, s$.

Proof. The system

$$
w_{i}^{\prime}(x, \lambda)= \begin{cases}d_{i} \lambda^{m_{i}} w_{j_{i}}(x, \lambda), & x \in\left[\eta_{0}, x_{0}\right], \quad i=q_{j}+1, \ldots, q_{j+1}, \\ 0, & x \notin\left[\eta_{0}, x_{0}\right], \quad i=q_{j}+1, \ldots, q_{j+1}, \\ 0, & i \notin q_{j}+1, \ldots, q_{j+1},\end{cases}
$$

is a minorant system to (4.7) in the interval $\left[\eta_{0}, 1\right]$, therefore

$$
\frac{p_{j}}{k_{j}} \leqslant \rho_{0}=\frac{p_{1}+\cdots+p_{s}}{k_{1}+\cdots+k_{s}}, \quad j=1,2, \ldots, s .
$$

If for some of the $j, p_{j}<\rho_{0} \cdot k_{j}$ then $\Sigma p_{i} / \Sigma k_{i}<\rho$ which contradicts (2.8).

Therefore, by the same consideration as before, it is enough to investigate one of these subsystems that means that we can investigate the system

$$
\begin{aligned}
& w_{j}^{\prime}(x, \lambda)= \begin{cases}d_{j} \lambda^{m_{3}} w_{j_{i}}(x, \lambda), & x \in\left[\eta_{0}, x_{0}\right], \\
0, & x \notin\left[\eta_{0}, x_{0}\right],\end{cases} \\
& w_{j}^{\prime}\left(\eta_{0}, \lambda\right)=b_{j}, \quad b_{j}>0, \quad j=1, \ldots, k_{1},
\end{aligned}
$$

and this system is irreducible.

9. Estimation of the solution of the minorant. We can rearrange the system (3.8), (4.8) and bring it to the normal form

$$
\begin{aligned}
w_{i}^{\prime} & =d_{i} \lambda^{m_{i}} w_{i+1}, \quad i=1, \ldots, k_{1}-1, \quad x \in\left[\eta_{0}, x_{0}\right], \\
w_{k_{1}}^{\prime} & =d_{k_{1}} \lambda^{m_{k_{1}} w_{1}}, \quad x \in\left[\eta_{0}, x_{0}\right], \\
w_{i}^{\prime} & =0, \quad x \notin\left[\eta_{0}, x_{0}\right], \quad i=1, \ldots, k_{1} .
\end{aligned}
$$

The solutions of the characteristic equation

$$
\mu_{i}=\omega \lambda^{\rho_{0}} e^{2 \pi(i-1) / k_{1}}, \quad \omega>0, \quad i=1, \ldots, k_{1} .
$$


It is obvious that the fundamental solution corresponding to $\mu_{i}$ is

$$
\begin{aligned}
& \tilde{W}_{i}(x, \lambda)=\left(1, \frac{\mu_{i}}{d_{1} \lambda^{m_{1}}}, \ldots, \frac{\mu_{i}^{k_{1}-1}}{d_{1} d_{2} \cdots d_{k_{1}-1} \lambda^{m_{1}+\cdots+m_{k_{1}-1}}}\right) \cdot e^{\mu_{1}\left(x-\eta_{0}\right),} \\
& \eta_{0}<x<x_{0}, \\
& \tilde{W}_{i}(x, \lambda)=\tilde{W}_{i}\left(x_{0}, \lambda\right), \quad x_{0}<x<1,
\end{aligned}
$$

therefore, any solution of the system (3.8), (4.8) is a linear combination of $W_{i}(x, \lambda)$, $i=1, \ldots, k_{1}$ :

$$
W(x, \lambda)=\sum_{i=1}^{k_{1}} c_{i} \tilde{W}_{i}(x, \lambda)
$$

We show that $c_{1}>0$ in (3.9).

Let us express in detail (3.9) at $x=\eta_{0}$, and let us use the fact that the initial condition (4.8) is a positive vector.

Hence, we get the following inequalities:

$$
\begin{gathered}
\sum_{i=1}^{k_{1}} c_{i}>0, \\
\sum_{i=1}^{k_{1}} \frac{c_{i} \mu_{i}^{k}}{d_{1} d_{2} \cdots d_{k} \lambda^{m_{1}+\cdots+m_{k}}}>0, \quad k=1,2, \ldots, k_{1}-1,
\end{gathered}
$$

as $d_{i}>0$ from (1.9) $\mu_{i}=\omega \lambda^{\rho_{o} \xi_{i}}, \omega \lambda^{\rho_{0}}>0$, for positive $\lambda$, we get the inequalities:

$$
\sum_{i=1}^{k_{1}} c_{i} \zeta_{i}^{k}>0, \quad k=0, \ldots, k_{1}-1 \text {, }
$$

where, $\zeta_{i}=e^{2 \pi(i-1) / k_{1}}, i=1, \ldots, k_{1}$.

Summing up all the inequalities of (4.9) we obtain

$$
\sum_{i=1}^{k_{1}} c_{i} \sum_{k=0}^{k_{1}-1} \zeta_{i}^{k}>0
$$

as

$$
\sum_{k=0}^{k_{1}-1} \zeta_{i}^{k}= \begin{cases}0, & i \neq 1 \\ k_{1}, & i=1\end{cases}
$$

It follows that

$$
\sum_{i=1}^{k_{1}} c_{i} \sum_{k=0}^{k_{1}-1} \zeta_{i}^{k}=k_{1} c_{1}>0
$$

therefore $c_{1}>0$.

LEMMA 5. For the solution of (3.8), (4.8) in the segment $\left[\eta_{0}, x_{0}\right]$, there are numbers $\omega>0, \beta, B>0$ such as, for $k=1,2, \ldots, k_{1}$,

$$
w_{k}(x, \lambda)> \begin{cases}B \lambda^{\beta} \exp \left(\omega \lambda^{\rho_{0}}\left(x-\eta_{0}\right)\right), & \eta_{0}<x<x_{0} \\ B \lambda^{\beta} \exp \left(\omega \lambda^{\rho_{0}}\left(x_{0}-\eta_{0}\right)\right), & x_{0}<x<1 .\end{cases}
$$


Proof. This is an immediate result of (2.9), (3.9) and (5.9):

$$
\begin{aligned}
0 & <w_{k}(x, \lambda)=\sum_{i=1}^{k_{1}} c_{i} \tilde{w}_{k i}(x, \lambda)=\sum_{i=1}^{k_{1}} \frac{c_{i} \mu_{i}^{k-1} e^{\mu_{1}\left(x-\eta_{0}\right)}}{d_{1} d_{2}, \ldots, d_{k-1} \lambda^{m_{1}+\cdots+m_{k}}} \\
& =c_{1} e^{\mu_{1}\left(x-\eta_{0}\right)} p(\lambda)(1+o(1)),
\end{aligned}
$$

where $c_{1}>0, p(\lambda)=\lambda^{\beta}(a+o(1))$ with real $\beta$ and $a \neq 0$; hence (6.9) follows for $k=1, \ldots, k_{1}$.

We get the same inequality for every subsystem of system (4.7), and by using the same reasoning as at the end of $\$ 6$ we get the following conclusion:

For the solution of (3.1), (4.1) there are numbers $\omega>0, \beta, B>0$ so that

$$
y_{n}(x, \lambda) \geqslant \begin{cases}B \lambda^{\beta} \exp \left(\omega \lambda^{\rho_{0}}\left(x-\eta_{0}\right)\right), & x \in\left[\eta_{0}, x_{0}\right], \\ B \lambda^{\beta} \exp \left(\omega \lambda^{\rho_{0}}\left(x_{0}-\eta_{0}\right)\right), & x \in\left[x_{0}, 1\right] .\end{cases}
$$

From (7.9) and (5.5) we get that there exist numbers $\omega>0, \omega^{*}>0, B>0$, $B^{*}>0, \beta, \beta^{*}$ so that

$$
\begin{array}{cl}
B^{*} \lambda^{\beta^{*}} \exp \left(\omega^{*} \lambda^{\rho_{0}}\left(x-\eta_{0}\right)\right) \leqslant y_{n}(x, \lambda)<B \lambda^{\beta} \exp \left(\omega \lambda^{\rho_{1} x}\right), & \eta_{0}<x<x_{0}, \\
B^{*} \lambda^{\beta^{*}} \exp \left(\omega^{*} \lambda^{\rho_{0}}\left(x_{0}-\eta_{0}\right)\right) \leqslant y_{n}(x, \lambda) \leqslant B \lambda^{\beta} \exp \left(\omega \lambda^{\rho^{\prime} x}\right), & x_{0}<x \leqslant 1,
\end{array}
$$

for real positive and large enough $\lambda$.

Conclusion. From the monotonity of the greatest characteristic root of nonnegative matrices and from (3.3), (4.3) and (2.5), (3.5), (4.5) and (1.9) we obtain that $\rho_{0} \leqslant \rho \leqslant \rho_{1}$; on the other hand from (1.7): $\rho_{0} \geqslant \rho_{1}$ hence $\rho_{0}=\rho=\rho_{1}$. From this together with (8.9) it follows that $y_{n}(1, \lambda)$ is an entire function of order $\rho$. In case $\rho$ is not an integer the equation $y_{n}(1, \lambda)=0$ has infinite sequence of solutions; this means that the system (3.1), (4.1), (5.1) has an infinite sequence of eigenvalues.

This completes the proof of Theorem 2.

10. Proof of Theorem 3. The proof of this theorem appears in [9]. For the sake of completeness we prove it again here, especially since that paper is hardly accessible for the English reader.

From (8.9) we obtain that, for $x=1$,

$$
B^{*} r^{\beta^{*}} \exp \left(\omega^{*} r^{\rho}\left(x_{0}-\eta_{0}\right)\right) \leqslant M\left(r, y_{n}\right) \leqslant B r^{\rho} e^{\omega r^{\rho}}, \quad r>1,
$$

where $B, B^{*}, \beta, \beta^{*}, \omega>0, \omega^{*}>0$ are constant.

In the circle $|\lambda|<r, r>1$, we will estimate the number of zeros $n(r)$ of the entire function $y_{n}(\lambda, 1)$ of finite order $\rho$, where $\rho$ is not an integer.

We will use the inequalities (see [5]):

$$
\begin{gathered}
n(r)<c \ln M(e r), \quad c>0 \\
\ln M(r)<T\left(r^{p} \int_{0}^{r} \frac{n(t)}{t^{p+1}} d t+r^{p+1} \int_{r}^{\infty} \frac{n(t)}{t^{p+2}} d t\right)
\end{gathered}
$$

where $T>0, p=[\rho]$.

From (1.10) it follows that

$$
n(r)<\sigma_{2} r^{\rho}, \quad \sigma_{2}>0 \text { is a constant. }
$$


If $n(t) \equiv 0$ in $0 \leqslant t \leqslant r$, then from (2.10)

$\ln M(r)<T\left(r^{p} \int_{r_{0}}^{r / q_{0}} \frac{n(t)}{t^{p+1}} d t+r^{p} \int_{r / q_{0}}^{q r} \frac{n(t)}{t^{p+1}} d t+r^{p+1} \int_{q r}^{\infty} \frac{n(t)}{t^{p+2}} d t\right)$,

$q$ and $q_{0}$ are constant numbers which we will choose later.

From (5.10) and (4.10) we get

$$
\begin{aligned}
\ln M(r) & <T\left(r^{p} \int_{r_{0}}^{r / q_{0}} \frac{\sigma_{2}}{t^{p+1-\rho}} d t+r^{p} n(q r) \int_{r / q_{0}}^{q r} \frac{d t}{t^{p+1}}+r^{p+1} \int_{q r}^{\infty} \frac{\sigma_{2}}{t^{p+2-\rho}} d t\right) \\
& \leqslant T\left[\frac{\sigma_{2} r^{\rho}}{(\rho-p) q_{0}^{\rho^{-p}}}+n(q r)\left(\frac{q_{0}^{p}}{p}-\frac{1}{p q^{p}}\right)+\frac{\sigma_{2} r^{\rho}}{(p+1-\rho) q^{p+1-\rho}}\right] .
\end{aligned}
$$

From the left inequality in (1.10) we obtain that there is such a number $\sigma$ that $\ln M(r)>\sigma r^{\rho}$ for $r>r_{0}$. Therefore (6.10) leads to

$$
\begin{aligned}
\ln M(r) & <T\left[\frac{\sigma_{2} \ln M(r)}{\sigma(\rho-p) q_{0}^{\rho-p}}+\frac{\sigma_{2} \ln M(r)}{\sigma(p+1-\rho) q^{p+1-\rho}}+n(q r)\left(\frac{q_{0}^{p}}{p}-\frac{1}{p q^{p}}\right)\right] \\
= & \ln M(r)\left[\frac{\sigma_{2} T}{\sigma(\rho-q) q_{0}^{\rho-p}}+\frac{\sigma_{2} T}{\sigma(p+1-\rho) q^{p+1-\rho}}\right]+n(q r)\left(\frac{q_{0}^{p}}{p}-\frac{1}{p q^{p}}\right) T .
\end{aligned}
$$

We will choose $q, q_{0}$ so large that

$$
\begin{aligned}
& A_{1}=\frac{\sigma_{2} T}{\sigma(\rho-p) q_{0}^{\rho-p}}+\frac{\sigma_{2} T}{\sigma(p+1-\rho) q^{p+1-\rho}}<1, \\
& A_{2}=T\left(\frac{q_{0}^{p}}{p}-\frac{1}{p q^{p}}\right)>0 .
\end{aligned}
$$

From (7.10) we get that

$$
n(r)>A_{3} \ln M\left(\frac{r}{q}\right)
$$

Here $A_{3}=\left(1-A_{1}\right) / A_{2}=$ const. From (4.10) and (8.10) we get the inequality $\sigma_{1} r^{\rho}<n(r)<\sigma_{2} r^{\rho}, r>1, \sigma_{1}>0, \sigma_{2}>0$ are constant. Therefore $\sigma_{1}\left|\lambda_{j}\right|^{\rho}<n\left(\left|\lambda_{j}\right|\right)=$ $j<\sigma_{2}\left|\lambda_{j}\right|^{\rho}$ holds, and so $a j^{1 / \rho}<\left|\lambda_{j}\right|<b j^{1 / \rho}, j=1,2, \ldots$, holds too when $a>0$, $b>0$ are constants independent of $j$.

Thus Theorem 3 is proved.

11. Concluding remark. Now we formulate the theorem we have mentioned in Remark 1.3.

We consider once more the problem (3.1), (4.1), (5.1). Let

$$
\mu_{j}(x, \lambda)=\psi_{j 0}(x) \lambda^{p_{j}(x) / q_{j}(x)}+\psi_{j 1} \lambda^{p_{j}(x)-1 / q_{j}(x)}+\ldots, \quad j=1,2, \ldots, n,
$$

be the solutions of the determinant

$$
\operatorname{det}\left|a_{i k}(x) \lambda^{m_{i}}-\delta_{i k} \mu\right|_{i, k=1}^{n}=0, \quad i, k=1, \ldots, n,
$$


and $\left(v_{j 1}(x, \lambda), v_{j 2}(x, \lambda), \ldots, v_{j n}(x, \lambda)\right)^{T}$ are the "eigenvectors" corresponding to $\mu_{j}(x, \lambda), j=1, \ldots, n$. (This means that the "eigenvectors" satisfy the equation:

$$
\sum_{k=1}^{n}\left(-a_{i k}^{1}(x) \lambda^{m_{i k}}+\delta_{i k} \mu_{j}\right) v_{j k}(x, \lambda)=0, \quad i=1, \ldots, n .
$$

We obtain that

$$
v_{j k}(x, \lambda)=\sum_{s=0}^{\infty} \psi_{j k s}(x) \lambda^{\left(p_{j k}(x)-s\right) / q_{j k}(x)}
$$

and

$$
\Delta=\operatorname{det}\left|v_{j k}(x, \lambda)\right|_{j, k=1}^{n}=\sum_{k=0}^{\infty} \phi_{k}(x) \lambda^{(p-s) / q} .
$$

These expansions are obtained in the neighbourhood of $\lambda=\infty$ and exist always for all $x$ for which $A(x, \lambda)$ appearing in (3.1) is bounded, where $q_{j}(x)>0$, $q_{j k}(x)>0, q(x)>0, p_{j}(x), p_{j k}(x), p(x)$, are integers.

All the expansions (1.11), (2.11), (3.11) are of the same form

$$
f(x, \lambda)=c(x) \lambda^{\alpha(x) / \beta(x)}+c_{1}(x) \lambda^{(\alpha(x)-1) / \beta(x)}+\ldots
$$

and we denote degree $f(x, \lambda)=\alpha(x) / \beta(x)$.

TheOREM 4. Let $A(x, \lambda)$ of (3.1), (4.1), (5.1) be a continuously differentiable matrix of $x: x \in[0,1]$ for every complex $\lambda|\lambda|<\infty$. Suppose the following conditions are satisfied:

(a) There exist $n$ continuously differentiable eigenvalues $\mu_{j}(x, \lambda), j=1, \ldots, n$, of the matrix $A(x, \lambda)$ of the form (1.11) with $p_{j} / q_{j}=$ const, $j=1,2, \ldots, n$, that is, all the functions $\psi_{j k}(x)$ of the expansion of $(1.11)$ are continuously differentiable and all the $p_{j}, q_{j}$ are constant and do not depend on $x$.

(b)

$$
\text { degree } \Delta=\max _{\left(j_{1}, j_{2}, \ldots, j_{n}\right) \in s_{n}}\left\{\operatorname{degree}\left[v_{1 j_{1}}(x, \lambda) \cdot v_{1 j_{2}}(x, \lambda), \ldots, v_{n j_{n}}(x, \lambda)\right]\right\}
$$

where $s_{n}$ is the set of all permutations of the numbers $1,2, \ldots, n$.

(c) All the numbers $p_{j} / q_{j}$ are positive and no one of them is an integer.

(d) All the numbers $\int_{0}^{1} \psi_{j 0}(x) d x, j=1, \ldots, n$, are different from zero and every two elements of the set $\left\{\lambda^{p_{j}} / q_{j} \int_{0}^{1} \psi_{j 0}(x) d x\right\}$ are different from each other for large enough $|\lambda|$.

Under these conditions there exists an infinite set of eigenvalues $\left\{\lambda_{j}\right\}$ of the problem (3.1), (4.1), for which

$$
\min _{1<j<n} \frac{p_{j}}{q_{j}} \leqslant \lim _{n \rightarrow \infty} \frac{\ln \left|\lambda_{n}\right|}{\ln n} \leqslant \varlimsup_{n \rightarrow \infty} \frac{\ln \left|\lambda_{n}\right|}{\ln n}<\max _{1<j<n} \frac{p_{j}}{q_{j}} .
$$

This theorem deals with a matrix $A(x, \lambda)$ the element of which may change their signs, not admitted in Theorem 2. We have formulated the last theorem in its simplest case for the sake of brevity, and postpone the proof and discussion of this theorem (and some others) because of its length. We intend to publish them in a separate paper. 


\section{REFERENCES}

1. G. A. Bliss, Algebraic functions, Amer. Math. Soc. Colloq. Publ., vol. 16, Amer. Math. Soc., Providence, R. I., 1933; Chapter 2.

2. Ju. E. Degutis and Sh. Strelitz, The existence of eigenvalues for a certain differential operator that depends on a parameter, Litovsk. Mat. Sb. 11 (1971), 535-556.

3. F. R. Gantmacher, The theory of matrices, vol. 2, Chelsea, New York, 1959.

4. M. V. Keldyš, On the characteristic values and characteristic functions of certain classes of non-self-adjoint equations, Dokl. Akad. Nauk SSSR 77 (1951), 11-14. (Russian)

5. B. Ja. Levin, Distribution of zeroes of entire functions, Transl. Math. Monographs, vol. 5, Amer. Math. Soc., Providence, R. I., 1964.

6. M. A. Naimark, Spectral analysis of non-selfadjoint operators, Amer. Math. Soc. Transl. (2) 20 (1962), 55-75.

7. I. G. Petrovski, Ordinary differential equations, Prentice-Hall, Englewood Cliffs, N. J., 1966.

8. Sh. Strelitz and Ju. E. Degutis, $A$ certain method of proof for the existence of eigenvalues for a certain differential operator with boundary conditions that depend on a parameter, Litovsk. Mat. Sb. 11 (1971), 683-690. (Russian)

9. Sh. Strelitz and V. Nekrašas, On existence of eigenvalues for a system of linear differential operator dependent on a parameter, Litovsk. Mat. Sb. 13 (1973), 191-209.

Department of Mathematics, University OF Haifa, Haifa, IsRael 